\title{
8. Ein Fragment mit mehrstimmiger Musik um 1470 in Basel
}

Um einen Zinsrodel des dem Reuerinnenkloster St. Maria Magdalena zu Basel zugehörenden Zehntausend-Ritter-Altars im Staatsarchiv Basel-Stadt, mit der Signatur Klosterarchiv St. Maria Magdalena HH, verwendet von 1492-1525, ist vorn und hinten ein papierenes Doppelblatt mit musikalischer Notation herumgelegt worden. ${ }^{1}$ Dessen vordere Doppelblattseite (vgl. Abb. 8a)) ist von einer neueren Archivars-Hand insgesamt rechts oben als fol. 1, die Doppelblatt-Rückseite dazu (vgl. Abb. 8b)) insgesamt als fol. 19 bezeichnet worden; der Rodel selbst umfaßt ein mit fol. 2-18' bezeichnetes Blatt-Corpus. Die beschriebene Folienzählung wird im Folgenden bei dem fraglichen Bruchstück insofern modifiziert verwendet, als die dort nebeneinander stehenden einzelnen Seiten in konventioneller Weise als fol. 1 und $1^{\prime}$ bzw. fol. 19 und $19^{\prime}$ bezeichnet werden, auch wenn, wie sogleich ausgeführt wird, diese Doppelseiten-"Hälften" heute sehr verschiedene Formate, keineswegs mehr die originalen aufweisen. Die Zählung erfolgt also grundsätzlich wie bei dem Zinsrodel, der von dem Musikfragment umschlossen ist. Dieses ist in neuerer Zeit vom Rodel-Corpus vorübergehend abgelöst worden; seine in dessen Mittelfalz auseinandergebrochenen „Hälften“ sind bei der darauf folgenden Restaurierung wieder verbunden, ,,in situm“ zurückgeführt und mit dem Rodel und einer angefügten neuen, allerdings nie genutzten Register-Blattfolge von neuem in jenen steifen Einbanddeckel eingefügt worden, der 1888 zur äußeren Befestigung des Ganzen zusätzlich angefertigt worden war.

Die hier beigegebenen Abbildungen halten das Bruchstück in ausgelöstem Zustand fest; dabei mißt die im Falz abgebrochene größere Blatthälfte von fol. 1/1' ca. 22,3 cm (Höhe) x ca. $16 \mathrm{~cm}$, die etwas kleinere von fol. 19/19' ca. 21,4 cm (Höhe) x ca.16,2 cm. Die Fuge, an der diese beiden Blatthälften aneinanderstoßen, ist allerdings, wegen der buchbinderischen Beschneidung am seitlichen Außenrand von fol. 19/19', mit der alten Mittelfalte des Doppelblattes nicht identisch: fol. 19/19' hat am äußeren Rand etwa die Hälfte eines Einzelblattes und am unteren Rand einen schmalen

1 Herrn Dr. Frank Labhardt sei für den Hinweis auf das vorliegende Fragment und seine Überlassung zur Veröffentlichung bestens gedankt. 
waagrechten Streifen verloren. Erheblicher Textverlust durch Buchbinderbeschneidung ist auch am oberen Rand des Doppelblatts eingetreten; von den Musikeintragungen her erscheint durchaus möglich, daß dieser Verlust sogar fast die Hälfte der ursprünglichen Blatthöhe beträgt, was dann ein großes originales Einzelblattformat von gegen $36 \mathrm{~cm}$ (Höhe) x $21,5 \mathrm{~cm}$ ergäbe (das Breitenformat ist aus dem wohl gut erhaltenen fol. 1/1' des Fragments leicht erschließbar). Da das Rodel-Corpus in zwei in ihrer Höhe leicht differierende Teile (fol. 2-10 und fol. 11-18) zerfällt und deren Formate eben den vom Buchbinder hergestellten Blattmaßen von fol. 1 bzw. fol. 19 entsprechen, da schließlich fol. 11, das erste Blatt des zweiten Rodel-Teils, mit 1521 oder 1522 datiert ist, können Bindung und alle die oben beschriebenen Buchbindereingriffe in das fragliche Deckblatt jedenfalls nicht vor diesem Zeitpunkt erfolgt sein - auch wenn die erste Eintragung des Rechnungsführers im Rodelcorpus, fol. 2 oben, ausdrücklich auf das Stiftungsjahr des Altars 1492 hinweist.

Rechnungsbelege sind, außer im Rodel selbst, auch auf dem Notenblatt, dort auf fol. 1, 19 und 19', angebracht; da sie offenkundig den durch Musikeintragungen nicht beschriebenen Raum nutzen, folglich nachgetragen sind und musikgeschichtlich nichts beibringen, können sie im Folgenden inhaltlich weitgehend übergangen werden. Das Papier des Blattes zeigt in fol. 19 das Wasserzeichen einer, wie Briquet sie benennt, ,tête humaine" mit dreifacher Nackenlocke: seine Provenienz ist norditalienisch oder südfranzösisch. ${ }^{2}$ Leider ist das Zeichen in eben der hier auftretenden Form sonst nicht dokumentiert; überdies tritt es, wie Briquet erklärt, zwischen etwa 1450 und 1535 in zahlreichen Varianten auf, so daß man am Wasserzeichen für eine sichere Papierdatierung leider keine Hilfe finden kann und sich deshalb vielmehr auf Beobachtungen an Manuskriptbild und Niederschrift verlassen muß, obgleich diese schließlich auch nur mäßig genaue Schätzungen gestatten.

Zunächst lassen die senkrecht ausgezogenen Linien zur seitlichen Begrenzung des Schriftfeldes und das in Musikmanuskripten des 15. Jahrhunderts aus deutschsprachigem Gebiet nicht seltene Fehlen jeglicher Mensurzeichen sowie die oft spitze Ausziehung von Oberlängen einzelner Textbuchstaben keine Zweifel daran, daß das Blatt jedenfalls in die zweite Hälfte des 15. Jahrhunderts zu setzen ist. Auch wenn der auf fol. 1 genannte Schuldner des Altars, „, her Rudolff seylor capplan zu lutenbach“, also ein Kloster-Kaplan Rudolf Seyler, in anderen Archivalien des Klosters schon

2 Vgl. Charles Moise Briquet, Les filigranes ..., Paris 1907, t. 2, Nr. 15670-15699. 
1447 und 1456 belegt ist ${ }^{3}$ und der erwähnte Namensvermerk auf dem Fragment, wie erwähnt, sicher erst nach vorhandener Musikeintragung niedergeschrieben worden ist, dürfte diese aber noch nicht um die oder bald nach der Jahrhundertmitte vorgenommen worden sein. Dies ergibt sich einerseits aus den Notenköpfen in den Musikeintragungen, die nur wenig von einer älteren, deutlich rhombischen Form bewahrt haben, vielmehr klar bereits zu einer elliptisch-rundlichen Gestalt tendieren. Gegen eine Datierung schon kurz nach Jahrhundertmitte spricht aber auch wenngleich dies kein absolutes Kriterium zu sein braucht - in den Notentexten die karge Zahl von Ligaturen, selbst in offenkundigen Mittelstimmen, und dabei, wenn überhaupt einmal auftretend, der alleinige Gebrauch von zweitönigen Ligaturen cum opposita proprietate. Schließlich empfehlen auch der in den Notenwerten eher ausgeglichene und relativ lineare Verlauf der erhaltenen Stimmen sowie die klare Zuordnung von Text und Musik im Beginn des Neujahrslieds auf fol. 1'/19 eine nicht allzufrühe Datierung innerhalb der zweiten Jahrhunderthälfte. Es wird darauf nochmals zurückzukommen sein, sobald der Inhalt des Bruchstücks genauer verzeichnet sein wird.

fol. 1 mit fol. 19', rechter Rand (vgl. Abb. 8a)): längerer Schlußabschnitt der T-Stimme einer nicht betitelten oder textierten und auch keinem Komponisten zugewiesenen Komposition, wegen ihrer Länge vielleicht eher einer Motette als eines Liedes.

$\mathrm{K}:-$

$\mathrm{Zu}$ der darunter folgenden Kurzzeile „Dem wirdigen Herrn“ folgen sogleich, zu fol. 1' mit fol. 19, linker Rand, weitere Erwägungen.

fol. 1' mit fol. 19, linker Rand (vgl. Abb. 8b)): Kurzer Schlußteil einer Dsowie eine ganze T-Stimme eines deutschen Neujahrsliedes ,Jch wuinsch der lieben gluick vnd hail//zů disem nuiwen anefang“.

$\mathrm{K}:-$

Der zitierte Liedtext, soweit erhalten, weist das Lied in die in der zweiten Hälfte des 15. Jahrhundert dichte und lebhafte Tradition von schriftlichen, auch gedruckten Neujahrsglückwünschen. Es ist zwar nicht beweisbar, aber danach auch nicht ausgeschlossen, daß die unfertige Bemerkung „Dem

3 Vgl. Emil A. Erdin, Das Kloster der Reuerinnen Sancta Maria Magdalena an den Steinen zu Basel von den Anfängen bis zur Reformation (ca. 1230-1529), Diss. Freiburg in der Schweiz, Freiburg Schweiz 1956, S. 159 (Seyler als Kaplan des ZehntausendRitter- und Elftausend-Mägdealtars). 
wirdigen Heren“ unterhalb des Satzbruchstücks auf fol. 1 mit fol. 19', rechter Rand (s. oben) die Anrede zu einem Neujahrswunsch darstellen sollte, wie er nun auch hier, also auf dem verso dazu, erscheint. Man kann sogar erwägen, ob hier ein Paar von Neujahrswunsch-Kompositionen faßbar werden könnte. Vgl. unten noch mehr dazu. - Die originale Stimmenzahl des Satzes ist unsicher; er dürfte drei-, vielleicht auch vierstimmig gehalten worden sein. Die fehlenden Parte müßen auf dem direkt auf fol. 1' folgenden Nachbar-recto gestanden haben.

fol. 19: Fragmentarische Textzeile ,...jam druiella leripon nun loß was ich dir sag die glog hat ..."; eben über den beiden letzten Wörtern sind noch zwei tiefgelegte, leider beschädigte Noten sichtbar, die vermutlich den Glockenschlag musikalisch nachahmen sollten. Diese kompositorische Absicht ist deshalb nicht völlig ausgeschlossen, weil diese Textzeile vom vierten Wort an offenkundig jenen Nachwächterruf eröffnet, der später, bezeugt vor allem seit dem 19. Jahrhundert, in der uns vertrauteren Form von „Hört ihr Herrn und laßt euch sagen ..." in zahlreiche Liederbücher eingegangen ist ${ }^{4}$ der hier vorliegende Beleg ist, soweit erkennbar, der frühste bisher erhaltene, der das Wächterrufen der nächtlichen Stundenzeit wohl auch mit der Warnung vor der nächtlichen Feuersgefahr kombiniert hat. ${ }^{5}$

4 Vgl. etwa Ludwig Erk/Franz M. Böhme, Deutscher Liederhort, Bd. 3, Leipzig 1893, S. 409f., Nr. 1580f.; zur Verbindung mit einer Choralmelodie von 1603 ebda.

5 Das Deutsche Volksliedarchiv Freiburg/Br., eine wissenschaftliche „Arbeitsstelle für internationale Volksliedforschung", laut e-mail- und Internetadresse angesiedelt bei der Universität Freiburg/Br., bei dem ich um Nachweis anderer früher Belege gebeten habe, war zu Auskünften nur nach Zahlung eines BearbeiterStundenlohns bereit, eine Voraussetzung, auf die ich aufgrund prinzipieller Überlegungen zur „Wissenschaftskultur“ nicht eingegangen bin. - Zu dem Nachwächterruf im Fragment fügt es sich, daß ausgerechnet für Basel eine besonders gründliche Studie vorliegt, die sich auch der Turmwächter dieser Zeit annimmt: Fritz Ernst, Die Spielleute im Dienste der Stadt Basel im ausgehenden Mittelalter (bis 1550), Diss. Basel 1945, in gleicher Form auch in der Basler Zeitschrift für Geschichte und Altertumskunde 45 (1945), S. 80-236. Danach war das Rufen der nächtlichen Stunden durch die Turmwächter Teil ihres Auftrags, vgl. ebda., S. 141f. Freilich bleibt die Art und Weise, wie dies zu geschehen hatte, in den Belegen unerwähnt, vgl. ebda., S. 151; der vokale Stundenruf geriet wohl mit dem Aufkommen der Basler Turmuhren seit dem späten 14. bis ins mittlere 15. Jahrhundert in Konkurrenz, wohl auch mit einem Stundenausruf, der instrumental ,gehornt" wurde, vgl. ebda., S. 142, auch Hans Jakob Nidecker, Von den Basler Turmbläsern, Privatdruck o. O. und J. <Basel 1971>, S. 13f. Da zwischen instrumentalem und vokalem Stundenausrufen in den Darstellungen oft nicht klar unterschieden wird, war die Mitteilung von Frau Silvia Siegenthaler, Stadtarchiv Brugg $(\mathrm{CH})$, dankenswert, wonach dort in den Stadtbüchern, wenngleich erst um 1500 belegbar, ausdrücklich festgelegt ist: „Vnd sol der wachter die stunden ruffen, des ersten by dem oberen tor ..."; es folgt die Nennung der weiteren Ruforte innerhalb der Stadt. 
Übrigens läßt auch Ludwig Senfl am Ende seines Liedes „Das Gläut von Speyer", gedruckt 1534 von Ott, die größte Glocke in tiefer Stimmlage erklingen. ${ }^{6}$

Die oben festgehaltenen drei Wörter am Zeilenbeginn bleiben unverständlich; selbst der Gedanke, sie seien, was in Musikhandschriften dieser Zeit zuweilen vorkommt, als Anagramm zu lesen, kann sie nicht klären (,nopirel alleiurd maj..., ,).

fol. 19, darunter: Fragmentarische Eintragungen von Anfang und Schluß eines Satzes „Puer nobis nascitur“ in D- sowie Anfang der zugehörigen, wohl der einstimmigen Vorlagemelodie ziemlich eng folgenden T-Stimme. Der Text gehört zu Weihnachten; am Seitenende folgen unterhalb des TBeginns längere Anfangspartien dreier Folgestrophen, mithin ,Jn presepe ponitur | sub feno asinorum | cognoverunt dominum | christum ...", „Tunc Herodes timuit | magno cum timore | infantes et pueros [?]| ...“ sowie „A et $\mathrm{e}$ et $\mathrm{i}$ et $\mathrm{o} \mid$ cantemus in choro| canticis et organis ben<edicamus?> ..."; mehr von diesen Textstrophen ist, wenn in dem Basler Manuskript überhaupt je vorhanden, verloren. Von diesem Text sind nicht wenige Parallelfassungen belegt, die freilich in Strophenbestand und Einzellesarten (wie schon hier) keineswegs immer übereinstimmen; soweit erkennbar, ist die sehr weit gestreute, also auch die musikalische ein- und mehrstimmige Gesamtüberlieferung dazu bisher nicht untersucht, so daß es geradezu verwegen wäre, hier eine stemmatisch-regionale Einordnung der im Basler Fragment vorliegenden Textversion vollziehen zu wollen. ${ }^{7}$ Damit bleibt es ganz ungewiß, ob der Schreiber einer Vorlage aus

6 Vgl. Ludwig Senfl, Deutsche Lieder, 2. Teil, hrsg. von Arnold Geering und Wilhelm Altwegg, Wolfenbüttel/Berlin 1940 (= EdM 15), S. 109-112 (Nr. 71), BaßStimme, passim. - Das fragliche Nachtwächterlied selber ist offenbar erstmals 1537 in Leipzig und mit einer Zahlung von 24 Groschen belegt, mit welcher der Rat der Stadt wohl dem Kantor Johannes Mopsus eine Anerkennung ausrichtete, ,von dem liedt hort ir hern lost euch sagen, der seiger der hat IX geschlagen“ wegen, vgl. Rudolf Wustmann, Musikgeschichte Leipzigs, Bd. 1, Leipzig/Berlin 1909, S. 37. Ein musikalisches Zitat aus dem Lied findet sich auch in einem vierstimmigen Satz Das erst Feuerbewaren von Leonhard Paminger, das Wolfgang Schmeltzels Sammlung Guter seltzamer und künstreicher teutscher Gesang sonderlich ettliche Künstliche Quodlibet ..., Nürnberg 1544, Nr. 23, bringt, vgl. Hans Joachim Moser, Tönende Volksaltertümer, Berlin 1935, S. 38f. - In der Nähe Basels, nämlich in den Mundartgedichten Wächterruf und Der Wächter in der Mitternacht des in Basel 1760 geborenen Johann Peter Hebel, ist das Lied wohl am frühsten, vor 1802/3, bezeugt, vgl. Johann Peter Hebels Werke, hrsg. von Wilhelm Zentner, Karlsruhe o. J., Bd. I, S. 137f., 146-149 und 365 (eben hier auch mit der beigefügten Vertonung ,eines Mannes von sehr gebildetem Geschmack“).

7 Herrn Kollegen Reinhard Strohm, Oxford, danke ich auch an dieser Stelle für den Hinweis auf mehrstimmige Fassungen bestens; zu einigen von ihnen vgl. Reinhard 
böhmischem Gebiet, aus einer norddeutschen Frauenzisterze oder aus dem Kreis der nordwesteuropäischen „Devotio moderna“ gefolgt ist - um nur einmal jene Provenienzgebiete zu nennen, an welche man bei dieser Art von paraliturgischen lateinischen Texten am ehesten denken möchte. Und für den hier vorliegenden, sicher drei-, vielleicht gar vierstimmigen musikalischen Satz - die restlichen Parte dürften auf dem vorangegangenen verso gestanden haben - waren keine Konkordanzen zu ermitteln.

$\mathrm{K}:-$

Auch wenn die Komposition mit einer Stimmenimitation von D und $\mathrm{T}$ beginnt, sollte man sie jedenfalls nicht für eine veritable Motette halten; dagegen spricht schon die zugehörige Reihe mehrerer in kurzer VierzeilerForm gehaltenen Textstrophen. Sie wird von der Forschung gelegentlich mit dem „Benedicamus domino“ verknüpft, in der Regel aber als „cantio“ bezeichnet und dann irgendwo zwischen artifizieller und einfacher, homophon-syllabisch deklamierender, manchmal auch volksliednaher Mehrstimmigkeit angesiedelt. Im vorliegenden Fall sollte man sich weniger von der heute noch erkennbaren Folge unterschiedlicher Stücke auf fol. 1' und fol. 19 irritieren lassen, da, wie sogleich zu zeigen sein wird, zwischen diesen beiden Seiten ziemlich viele weitere Blätter und Musikeintragungen gestanden haben dürften; etwas unsicherer könnte man im Blick auf den direkten Anschluß von „Nachtwächterruf“ und „,cantio“ auf fol. 19 werden; allerdings sind Musik-Sammelhandschriften dieser Zeit aus deutschsprachigem Gebiet nicht immer sehr skrupelvoll in der Reihung sehr verschiedener Kompositionen gewesen.

fol. 19': s. oben, zu fol. 1.

Es mag sich nach der Sichtung des Inhalts empfehlen, nochmals kurz zur äußeren Gestalt des Bruchstücks zurückzukehren und hier bei einer noch nicht berührten Notiz auf fol. 19' unten zu verweilen. Man liest dort die vier Zeichen „1o24“. Die Notiz kann nicht vom Rechnungsführer der Rodelaufzeichnungen stammen, da sie keine Summe von Beträgen darstellt, die aus den voranstehenden Rechnungsbelegen resultiert, und da

Strohm, Das Orationale Kaiser Friedrichs III. und das europäische geistliche Lied, in: Wiener Quellen der älteren Musikgeschichte zum Sprechen gebracht. Hrsg. von Birgit Lodes, Tutzing 2007, S. 229-256. - Übrigens ist das fragliche Stück auch sonst in Basler Manuskripten erhalten, die aber keine Noten bieten oder, weil ,,in gotischen Choralnoten" überliefert, nicht leicht vergleichbar sind; vgl. Frank Labhardt, Das Cantionale des Kartäusers Thomas Kreß. Ein Denkmal der spätmittelalterlichen Musikgeschichte Basels, Bern/Stuttgart 1977 (= Publikationen der Schweizerischen Musikforschenden Gesellschaft), S. 238. 
auch ihre Schreiberhand mit derjenigen jener Belege nicht übereinstimmt. Sie muß deshalb von dem Musikschreiber herrühren, und unter dieser Voraussetzung gewinnt sie sogleich ihren Sinn: Noten- und Textkopisten der fraglichen Zeit pflegen gerne, entweder auf der jeweils ersten oder der letzten Seite einer Manuskriptlage, eine Angabe anzubringen, die dem Buchbinder anzeigen soll, in welcher Reihenfolge die Lagen einer Sammelhandschrift einzureihen seien und/oder wie umfangreich eine Lage sei: dies war bei Handschriften mit, einem Buchbinder schwer verständlichen Musiknotationen besonders nötig. Im vorliegenden Fall hat man offenbar mit einer Marke auf einem ersten Außenblatt einer Lage zu rechnen; „1o24“ könnte, modern übertragen, soviel wie „1. [oder: primo] 24“ heißen, also ein erste Lage mit 24 Blättern anzeigen. Danach hätte in dieser Lage zwischen fol. 1' und fol. 19 noch eine erhebliche Zahl, nämlich eine solche von 22 beschriebenen Blättern bzw. 11 Doppelblättern, gelegen, von denen heute freilich allesamt verloren sind. Fol. 19' ${ }^{\prime}$ ist in seiner Originalgestalt offenbar unbeschrieben geblieben, was bei äußeren Schlußblättern von Lagen in einem Musikmanuskript jener Zeit nicht selten ist; da oft nicht alle Stimmen einer Komposition auf ein solches (halbes) Schlußblatt bzw. eine einzige Schlußseite paßten, hat man dieses bzw. diese, selbst wenn weitere Lagen angefügt wurden, häufig leer gelassen, allerdings dann gerne nachträglich noch kleinere Füll-Kompositionen darauf notiert. Da im vorliegenden Fall die erwähnte Lagen-Numerierung nur Sinn macht, wenn noch weitere Lagen angereiht werden sollten, darf man schließen, daß das Provenienzmanuskript noch weitere Lagen, also einen respektablen Gesamtumfang besaß. Sein Verlust ist danach besonders bedauerlich.

Leider ist das deutsche Wortmaterial in dem Fragment nicht so reichhaltig, daß eine eindeutige Bestimmung dialektaler Eigenheiten und damit eine geographisch-regionale Verortung des Bruchstücks möglich wäre. Aber es läßt sich, nach Auskunft eines Fachmannes, ${ }^{8}$ doch ,sagen, daß einer Einordnung ins Alemannische auch nichts widerspricht. Vor allem das erhaltene lange , ̈̈“ in „nuiwen“, in Kombination mit dem ,ai““ in „hail“ und der Erweichung des Auslautes in „glog“, kann man auch als positive Indizien für das Niederalemannische werten“. Die von hier aus unternommene Recherche nach Konkordanzen im Buxheimer Orgelbuch - das ja nach Erkenntnissen der letzten Jahre ja nicht in bayerischem, sondern in

8 Herrn Kollegen Klaus Grubmüller, Göttingen, sei auch hier bestens für seine Beurteilung gedankt. 
alemannischem Gebiet entstanden sein dürfte, ${ }^{9}$ und dies sogar noch etwa zu gleicher Zeit wie das vorliegende Bruchstück - ist allerdings negativ ausgegangen, wobei aber keineswegs klar ist, ob dies nur in einer durchgehenden Repertoireverschiedenheit oder in einer regionalen Ungleichheit begründet ist. $\mathrm{Zu}$ der vermuteten Basler Provenienz könnte übrigens auch das Auftreten des Neujahrsliedes passen: es scheint nämlich so zu sein, daß die Neujahrswunsch-Praxis, wie sie sich im vorgerückten 15. Jahrhundert vor allem auf Einblattdrucken - meist mit dem Wunsch „Ein gut selig ior“ - , auf Kalendern bzw. Almanachen, auf Zeichnungen und in Gedichttexten äußert, in Basel besonders gerne praktiziert worden ist; ${ }^{10}$ sie lebt in diesem Gebiet sogar noch im 18.-20. Jahrhundert in den „Neujahrsblättern“ verschiedener Basler, übrigens auch Zürcherischer Gesellschaften weiter.

So wie sich die Verhältnisse schließlich präsentieren, möchte man zu Herkunft und Alter vorläufig festhalten: das Fragment, Rest einer Sammelhandschrift von einigem Umfang, dürfte aus Basel oder aus dem nähern Umkreis der Stadt stammen. Vielleicht jünger als München 810, das Schedelsche Liederbuch, - und auch von entschieden größerem Blattformat als jenes - , aber doch gewiß älter als etwa Augsburg 25, das Augsburger Fragment, ${ }^{11}$ dürfte das Bruchstück am ehesten in die siebziger Jahre des 15. Jahrhunderts gehören. Aufgrund dieser Datierung kommt ihm, trotz seinem geringen Umfang, ein besonderes Interesse zu: seine Entstehung fällt jedenfalls in einen Zeitraum, in dem eine süddeutsche Liedüberlieferung nicht besonders gut dokumentiert ist.

9 Vgl. Lorenz Welker, Musik am Oberrhein im späten Mittelalter. Die Handschrift Strasbourg, olim Bibliothèque de la ville, C. 22, Hab.schr. Basel (maschr.) 1993, Bd. I, S. 212-217.

$10 \mathrm{Vgl}$. etwa Hans Koegler, Einige Basler Kalender des XV. und der ersten Hälfte des XVI. Jahrhunderts, Anzeiger für Schweizerische Altertumskunde, N. F. 9 (1909) [erschienen 2010], S. 153-169, 235-246 und 330-249, zur Stellung des Wunsches bes. S. 156.

11 Vgl. Das Liederbuch des Dr. Hartmann Schedel. [Faksimile]. Mit einem Vorwort von Bettina Wackernagel, Kassel etc. 1978 (= EdM 84), sowie Martin Staehelin, Das Augsburger Fragment. Eine wenig beachtete süddeutsche Quelle zur mehrstimmigen Musik des späten 15. und des frühen 16. Jahrhunderts, Augsburger Jahrbuch für Musikwissenschaft 1987, Tutzing 1987, S. 7-63. 